\title{
Per umbram fons ruit. \\ Petrarca in Elicona. Paesaggio e Umanesimo
}

\section{Rossend Arqués}

Universitat Autònoma de Barcelona

\section{Abstract}

In questo articolo che fa parte di una ricerca sul paesaggio in Petrarca, si analizzano la sua esperienza personale e la sua concezione del paesaggio della solitudine nonché l'importanza che esso ha avuto nella sua creazione letteraria e nella costruzione della figura ideale dell'intellettuale umanista che tanta influenza avrà in seguito. Qui l'autore compie un tentativo di lettura degli elementi iconografici del disegno autografo del manoscritto contenente la Historia naturalis di Plinio dietro ai quali si nasconde non solo il nome del poeta ma anche la sua mitografia umanistica.

Parole chiave: Petrarca e il paesaggio, Historia naturalis di Plinio, Valchiusa, solitudine, umanesimo.

\section{Abstract}

This article, forming part of broader research into landscape in the literature of Petrarch, analyses the personal experience of the poet and his conception of the landscape of solitude, as well as the role that this has played both in the writer's literary creation and in the construction of the ideal figure of the humanist intellectual, later to be of such influence. The author proposes a reading of the iconographic elements of the autographic design contained within Pliny's Historia naturalis, behind which there lies not only the poet's name but also his humanistic mythography.

Key words: Petrarch and landscape, Pliny's Historia naturalis, Vaucluse, Familiares, solitude, humanism.

1.

Quando l'uomo ha smesso di contemplare alberi, montagne, fiumi e campi nella loro dimensione naturale per trasformarli in elementi di un paesaggio, per sottoporli a un processo di spiritualizzazione? Quale fu l'istante in cui è avvenuta questa trasformazione estetica? Fino a che punto Francesco Petrarca, il grande capostipite dell'Umanesimo, è anche il responsabile della crea- 
zione del paesaggio come categoria estetica, intellettuale e morale, grazie alla quale la natura, convertendosi in concetto, è diventata colonia dello spirito umano? Possiamo parlare di Petrarca come del creatore del paesaggio e, se così fosse, in quali termini? In realtà già l'uomo delle caverne, quando dipingeva mandrie di bufali o di cervi accerchiate da cacciatori armati di archi e frecce, sicuramente stava investigando nuovi campi poetici attraverso dei segni estetici molto stilizzati. Si tratta, dunque, di capire esattamente che posto ha Petrarca nella storia del paesaggio letterario e artistico. Ma dato che questo obiettivo costituisce il logico processo finale della mia analisi delle diverse forme e funzioni e dei diversi valori del paesaggio nel poeta, qui mi riprometto soltanto un approccio a uno di questi aspetti, forse il più centrale per il futuro concetto di luogo dell'umanista - e in generale dell'intellettuale, nel mondo: cioè, lo scenario nel quale e grazie al quale è possibile una vita intellettuale piena, libera e creativa, in quanto luogo dove lo spirito può trovare se stesso e nel quale, specificamente per Petrarca, potevano manifestarsi sia la divina armonia del cosmo che la disarmonia del mondo materiale.

Prima di lui il luogo della contemplazione, della creatività, del pensiero e della poesia era soprattutto la camera (Dante, Vita nova ${ }^{1}$ e anche Petrarca $R V F)^{2}$, lo studio o la cella, nel caso dei monaci. Petrarca, invece, pur non cessando di riconoscere il valore della stanza — d'altronde che cos'è Valchiusa se non una stanza aperta agli elementi naturali, trasformata in schermo sui cui proiettare i propri fantasmi? - - estende la sfera dell'interiorità anche ai paraggi della sua casa, come in parte avevano già fatto quelli stessi eremiti di cui egli stesso si occuperà nel De vita solitaria (DVS) e nel De otio religioso (DOR).

Qui ci interessa ripercorrere i punti centrali dell'esperienza e della concezione petrarchista del paesaggio della solitudine e l'importanza che ha avuto nella sua creazione letteraria e nella costruzione della figura ideale dell'intellettuale umanista che tanta influenza avrà in futuro. ${ }^{3}$

1. Dante, Vita Nuova cap. 14, par. 9, «e ricorsi a lo solingo luogo d' una mia camera (cap. 3, 3); «Avvenne quasi nel mezzo de lo mio dormire che me parve vedere ne la mia camera lungo me sedere uno giovane vestito di bianchissime vestimenta.[cap. 12, par. 3] [...] «e anzi ch' io uscisse di questa camera, propuosi di fare una ballata, ne la quale io seguitasse ciò che lo mio segnore m' avea imposto" [...]; E partitomi da lui, mi ritornai ne la camera de le lagrime; ne la quale, piangendo e vergognandomi, fra me stesso dicea: «Se questa donna sapesse la mia condizione, io non credo che così gabbasse la mia».

2. $R V F$ 234, 1-5: «O cameretta che già fosti un porto / a le gravi tempeste mie diurne, / fonte se' or di lagrime nocturne, / che 'I dì celate per vergogna porto. // O letticciuol che requie eri et conforto». Cfr. Andrea ZANZOTTO, «Petrarca fra il palazzo e la cameretta», in Scritti sulla letteratura. Vol. I: Fantasie di avvicinamento, Milano: Oscar Mondadori, 2001, p. 261-271.

3. H. FrIEDRICH, Petrarcas Landschaften. Zur Geschichte äesthetischer Landschaftserfahrung, in "Schriften und Vorträge des Petrarca-Instituts», XXIX, Köln, Krefeld, 1979; Bernhard KÖNIG / TRIER, Petrarcas Landschaften. Philologische Bemerkungen zu einer neuen Deutung, in «Romanischen Forschungen», (1980), 92, 3, p. 251-282. 


\section{2.}

Il luogo della creazione artistica e intellettuale è più che mai fondamentale. È di conseguenza necessario sceglierlo con cura affinché possa produrre gli effetti desiderati nello spirito dello scrittore, dell'umanista. Di questo Petrarca ha piena coscienza. Ciò nonostante si trova davanti a una dicotomia, causata, come tante altre, dalla novità del suo pensiero laico. Egli esalta la bellezza della natura in contrasto con la tradizione del modello dell'eremita (Sant'Antonio, per esempio), da lui non soltanto conosciuto e studiato, ma anche rivendicato nel libro II del $D V S$, il quale rifuggiva da questa bellezza in quanto nemica della contemplazione e della preghiera. Ora, invece, il luogo che Petrarca sceglie per meditare e realizzare la sua opera, non è affatto un deserto, bensì un locus amoenus in cui il desiderio umano [o la volontà umana] potrebbe anche essere fuorviata dallo spettacolo di tanta lussureggiante vegetazione. È innegabile che i monaci e soprattutto gli asceti per lui rappresentano il modello assoluto di comportamento di vita solitaria. Anche se, forse, si dovrebbe dire rappresenterebbero, poiché riconosceva che le vite di questi uomini e donne erano per lui irraggiungibili e non solo a causa della sua proverbiale mancanza di volontà e di decisione, per colpa della quale è incapace di far proprie le ferme decisioni del fratello Gherardo ${ }^{4}$ e di tutti coloro che come lui hanno scelto, senza il minimo tentennamento, di abbracciare la vita monacale; ma anche perché la sua idea di isolamento dal mondo è completamente diversa da quella del fratello e dei cenobiti della tradizione cristiana. Egli si sente più vicino alla solitudine problematica, umana e laica espressa nelle lettere dei suoi amati Seneca e Cicerone.

4. Fam. XV 4, 10-16: «Ma che fare? mi creda chiunque ha fiducia in me: se sotto il cielo mi fosse dato trovare un luogo qualunque non dirò buono, ma non cattivo, o almeno non pessimo, volentieri e per sempre mi fermerei; ma ora come in un duro giaciglio io mi volto e mi rivolto, né con tutta la buona volontà riesco a trovare il bramato riposo; e così alla mia stanchezza, non potendo con la morbidezza del letto, provvedo col continuo mutare; vada vagando e sembro un eterno viandante. Quando sono stanco della durezza di un posto mi reco in un altro, che non è meno duro, ma cui la novità diminuisce per un poco la durezza. Così io sono sbattuto qua e là, pur sapendo che nessun luogo sulla terra è tranquillo, ma che attraverso a molti travagli si deve anelare alla quiete [...] Lo ripeto, sono malato, e si vede anche se non lo dico; se fossi sano, mi comporterei con più fortezza; ma non per questo il mio lettuccio diventerebbe morbido e e piano» (alla sorgente della Sorga, il 26 di febbraio [1346-47]), (Sed quid agam? credat quisquis unquam michi aliquid crediturus est: siquem ego sub celo locum bonum, imo non malum, ne dicam pessimum, reperirem, cupide perseveranterque subsisterem; nunc velut in preduro recubans grabatulo, huc illuc versor, nec votis omnibus quesitam requiem invenio; itaque lassitudini mee, quia cubilis suavitate non possum, alternatione subvenio; vagor igitur et sine fine peregrinus videor. [11] Dum enim loci huius duritie fessus sum, in alterum feror, etsi nichilo molliorem, cuius tamen interim novitate saltem leniatur asperitas. Sic iactor non ignarus nullum hic quietis locum, sed per multos labores ad requiem suspirandum, hic vero perpetuo laborandum gemendumque [...] Iterum dico, quod tacito me apparet: eger sum; fac me autem sanum, feram fortius; sed non ideo mollis aut equus lectulus meus erit - lectulus vite huius in quo fessus iaceo-, quin potius asper inamenus immundus iniquus scrupulosus et qui sanissimos etiam vehementer exagitet), cit. per Francesco Petrarca, Opere, a cura di Mario Martelli, Milano: Sansoni editore, 1975. 
Ma oltre a fornirci la prima enciclopedia degli uomini solitari (per la maggior parte cristiani), ${ }^{5}$ il primo libro del $D V S$ ci offre anche riflessioni sistematiche sull'esperienza della solitudine, i cui modelli teorici non sono tuttavia cristiani, almeno non esclusivamente, ma anzi essenzialmente pagani. Ciò è chiaramente evidente nell'affermazione che «la solitudine senza cultura è certo, un esilio, un carcere, una tortura. Aggiungivi la cultura: diventa la patria, la libertà, il godimento». E a supporto di questa tesi aggiunge: «A proposito dell'ozio, è celebre quel detto di Cicerone: "Cos'è più dolce di un ozio dedicato alle lettere!". Di rimando, non meno famose quelle parole di Seneca: "Un ozio senza lettere è la morte, è il funerale di un vivo"». ${ }^{6}$ Più avanti ricorda quanto Cicerone e Virgilio fossero amanti dell'isolamento, e come il primo in ogni circostanza, ma particolarmente "quando si accingeva a lavorare al trattato delle leggi civili, cercava querce frondose e piacevoli recessi e rive ed ombra [...], e pioppi altissimi, e musica di uccelli, e gorgogliare di acque correnti, e un' isoletta posta proprio in mezzo al fiume in modo da dividerlo in due parti uguali», il secondo, invece, «apprestandosi a cantare in un carme bucolico il suo Alessi [...], si aggirava solo fra i monti e le selve, recandosi spesso tra i folti faggi, ombrose vette» (DVS I VII). Non gli sfugge che tali comportamenti sono in contrasto con l'affermazione di Quintiliano, secondo la quale l'uomo che deve scrivere ha bisogno necessariamente di un «luogo appartato e solitario e di una calma altrettanto profonda», anche se non «in mezzo alla natura». ${ }^{7}$ I boschi e le foreste non possono essere utili a questo scopo proprio perché la loro bellezza distrae lo spirito, il quale non può essere soggetto a due stimoli diversi; «talché mi sembra — conclude Quintiliano— che questo piacere rallenti piuttosto che tendere l'attenzione».

Davanti a questa presa di posizione, molto diffusa tra gli scrittori cristiani e di cui troviamo eco nella Fam. XIII $8,{ }^{8}$ Petrarca non troverà nessun altro

5. Carlo Delconno, Petrarca e l'agiografia dei «solitari», "Lettere italiane» (2005), n. 3, p. 367-390 y también Manlio PASTORE STOCCHI, «Divagazione su due solitari: Bellerofonte e Petrarca», in Da Dante al Novecento. Studi critici offerti dagli scolari a Giovanni Getto nel suo ventesimo anno di insegnamento universitario, Milano: U. Mursia, 1970; Carlo OssolA, Verbum et secretum (Des Pères de l'Eglise et de Pétrarque), "Versant», III (1982), p. 23-43; W. SCOTt Blanchard, Petrarch and the Genealogy of Ascetism, in "Journal of the History of Ideas», 62, 2001, p. 401-423.

6. "Otium sine literis mors est, et hominis vivi sepultura [...] Nam de otio quidem illud Ciceronis notum "Quid dulcius otio literato?". Contraque, non minus illud Senecas vulgatum: "Otium sine literis mors est, et hominis vivi sepultura" ", Francesco PETRARCA, De Vita solitaria, I III, in ID, Opere latine, a cura di Antonietta Bufano, vol. I, Torino: UTET, 19.

7. «dum alter cum sepe alias tum presertim ad tractatum legum civilium accessurus, frondosas quercus et delectabiles secessus, quoque ibi scriptum memini ripa et umbram'», et procerissimas populos, et concentum avium, et strepitum fluviorum, atque equas in partes scissi amnis in medio isulam parvam et huic nostre simillimam querit; alter autem suum Alexim, quisquis is est, pastorio carmine laudaturus, «inter densas umbrosa cacumina fagos» assidue veniens, solus in montibus et silvis id faceret», Francesco PeTrarCA, De vita solitaria, cit., Libro I VII.

8. «Passo l'estate al fonte della Sorga [... Ho dichiarato guerra al mio corpo. Così mi aiuti quel Dio, senza il cui aiuto sarei vinto, come è vero che la gola, il ventre, la lingua, le orecchie, gli 
miglior fondamento che la propria esperienza; e senza pretendere, almeno formalmente, di farla passare come legge generale di vita per tutta l'umanità, è evidente che egli si presenta come il fondatore di un nuovo tipo di relazione tra l'intellettuale e la natura.

Sebbene io abbia esperimentato che in nessun luogo il mio ingegno è più fecondo e più pronto che nelle selve e fra i monti, e che ivi, se pur mi è dato in qualche luogo, mi vengono in mente pensieri magnifici e parole adeguate e rispondenti ai concetti, non vorrei tuttavia fare una legge generale di quella che è forse una mia particolarità.?

Tutta la sua riflessione non conduce che a una conclusione verso la quale lo avevano già avviato alcune riflessioni del prediletto Agostino del De doctrina cristiana:

Ecco il portico, ecco la sede che l'uomo santo ed eloquente desidereva: viti, tralci, fronde, canne, e fra queste la solitudine sempre gradita agli studiosi ${ }^{10}$

\section{3.}

Nella Seniles X 2, testo autobiografico complementario a Posteritati, scritto durante il suo soggiorno a Venezia verso la fine del 1367 nel quale rifa tutto il percorso della sua vita, egli ricorda la prima volta che vide Valchiusa, il luogo magico che diventerà il centro della sua vita intellettuale e poetica per moltissimi anni. Dopo aver lasciato Carpentras con Guido Sette, suo amico d'infanzia nonché futuro vescovo di Genova, e lo zio di questi, alla ricerca di «cose nuove» visita le sorgenti della Sorga, un affluente del Rodano, che già allora era meta turistica, come egli stesso spiega. Qui fu tanto rapito dalla bellezza del posto che

occhi non mi sembrano spesso parti del mio corpo, ma empi nemici. Di molti mali ricordo ch'essi mi furon cagione, e soprattutto gli occhi, che mi spinsero sempre verso la rovina. Io li ho chiusi in modo che non vedano altro che il cielo, montagne e fonti, e non oro o gemme o avorio o porpora, non cavalli, salvo due e assai meschini, che trasportano me e il mio unico servo per queste valli [...] Un vento disperse tutta quella dolcezza; ora non odo altro che rari muggiti di buoi o belati di pecore o canti di uccelli, e il perenne mormorio delle acque» [Ad fontem Sorgie estatem ago [...]. Corpori meo bellum indixi. Ita me Ille adiuvet sine cuius ope succumberem, ut gula ut venter ut lingua ut aures oculique mei sepe michi non artus proprii sed hostes impii videntur. [2] Multa quidem hinc michi mala provenisse memini, presertim ab oculis, qui ad omne precipitium mei fuerunt duces. Hos ita conclusi ut preter celum preter montes ac fontes fere nichil videant, non aurum non gemmas non ebur non purpuram non equos, nisi duos eosque ipsos exiguos, qui cum unico puero his me vallibus circumvectant], Francesco PeTrarCA, Opere, cit.. Cfr. anche Epystole metriche, I 8 e III 3.

9. «Itaque quamvis ego nusquam felicius quam in silvis ac montibus ingenium experiar, nusquam michi paratius et magnifici sensus occurrant — sicubi tamen magnificum aliquid occurrit - et equa concetibus verba respondeant, nolim tamen, quod michi forsan singulare est ad universos trahens», Francesco PetrarCA, De vita solitaria, cit., Libro I VII.

10. «Ecce quam porticum et quam sedem vir sanctus et eloquens appeteba: vites, palmites, frondes, arundines, et inter haec studiosis semper amabile secretum», Francesco PeTraRCA, De vita solitaria, cit. Libro I VII. 
in quello stesso momento decise non soltanto che sarebbe ritornato a visitare una tale meraviglia ma che vi avrebbe fissato dimora, preferendola a qualunque città per quanto famosa e importante («magnis urbibis prelaturus sim!»). ${ }^{11}$ Quel luogo era infatti il più adatto alla sua indole più intima e il tempo che vi trascorse fu così lieto che "sempre» lo fece coincidere con la miglior epoca della sua vita, addirittura come l'unica sua vera e autentica vita. Qui, come in molte altre occasioni, non manca di sottolineare che mentre gli uomini pubblici, compreso lo stesso amico e confessore Guido Sette, errano privi di libertà nei palazzi del potere e per le strade cittadine sfiorando appena tanti altri indaffarati come loro, egli passeggia tranquillo e solitario ${ }^{12}$ tra i boschi, disprezzando le ricchezze inseguite dagli altri, nella quiete campestre formata dal chiaro mormorio del torrente, dal muggito dei buoi che risuona nella valle e dal canto degli uccelli che intonano tra le fronde degli alberi concerti di notte e di giorno. ${ }^{13}$ Lì trova rifugio al caos cittadino e un luogo così poco esposto ai pericoli del mondo da poter passeggiare per campi e montagne senza paura di essere aggredito da animali o persone («ubi in montibus noctu solus ac securus erraverim!»). Il poeta è così convinto che là non esistono nemici che definisce quel luogo un'eterna oasi di pace («illum immobilem ac pacificum locum fore»).

Ciò nonostante, anche quell'oasi idilliaca verso la metà del 1350 sembra ridursi solo a un bel ricordo, da quando il mondo si è volto di male in peggio. Tutta la lettera di contenuto autobiografico indirizzata a Guido Sette ha come motivo di sfondo l'amara constatazione che la decadenza interessa ormai ogni angolo non soltanto della cosiddetta civiltà ma anche della natura, senza purtroppo eccezione di Valchiusa, ora preda dei lupi che fanno strage di agnelli, e di ladroni che assaltano e saccheggiano le case con una avidità tale che risulta impossibile difendersi, per quanto alte siano le protezioni erette. Anche quel porto sicuro, descritto in tanti passi come rifugio inespugnabile ( Hec tibi quoque, Sorgie ad fontem, quo rursus e tanto Italie naufragio velut in portum fugi, et preteriti dolens et venturi trepidus, dictabam Kalendis Sextilibus, intempesta nocte», Fam. VI 5,18$),{ }^{14}$ è stato spazzato via dall'onda gigantesca della modernità.

11. «"Ecco', dissi, 'un luogo veramente adatto all'indole mia, che se potrò, vorrò un dì preferire a qualsivoglia più famosa città" " [En nature mee locus aptissimus, quemque, si dabitur aliquando, magnis urbibus prelaturus sim!], Francesco PETRARCA, Le senili, a cura di Guido Martellotti, Torino: Einaudi, 1976.

12. Fam. IV 4, 5: "me vagante solitario nelle selve, e la sera nei prati lungo le rive della Sorga» [me solivagum mane in silvis, sero autem in pratis, Sorgie ripis obambulantem invenerunt mei], Francesco Petrarca, Opere, cit.

13. Sen. X 2: "que me, mirum dictu, solitarium contemptorem, profugum in media silvas, usque ad invidiam insecute sunt. Quid vero tibi nunc ego illud agreste silentium, illud nitidissimi amnis assiduum murmur, illum boum sonoris in vallibus mugitum, illos volucrum in ramis non diurnos modo concentus sed nocturnos explicem? Scis omnia, etsi me hac in parte non penitus sequi ausus, quotiens tamen urbanis tumultibus te furari posses - quod perrarum erat - cupide illuc, ut e procellis in portum fugire solitus", Francesco PETRARCA, Le senili, cit.

14. Fam. VI 5, 18: «dalla sorgente della Sorga, dove un'altra volta io mi son rifugiato, come in un porto, da tanto naufragio della nostra Italia, dolente del passato e trepido dell'avvenire» 
La metafora del porto applicata a Valchiusa ricorre con una certa frequenza, soprattutto nelle Familiares. È il suo abituale riparo durante le tempeste dello spirito (Fam. V 1 e XIII 6), e in esso trova ricetto dopo il naufragio della sua Italia «dove un'altra volta io $\mathrm{mi}$ sono rifugiato come in un porto, da tanto naufragio della nostra Italia, dolente del passato e trepido dell'avvenire» ${ }^{15}$ (Fam. VI 5 18). E ancora, la Fam. XI 4 inviata a Filippo, vescovo di Cabassoles con data 27 giugno 1351, ne contiene un'infiammata esaltazione e il fermo proposito di volervi risiedere fino all'ora estrema:
Valle locus Clausa toto michi nullus in orbe
Gratior aut studiis aptior ora meis.
Valle puer Clausa fueram iuvenemque reversum
Fovit in aprico vallis amena sinu.
Valle vir in Clausa meliores dulciter annos
Exegi et vite candida fila mee.
Valle senex Clausa supremum ducere tempus
Et Clausa cupio, te duce, Valle mori. ${ }^{16}$

Ancora agli inizi degli anni cinquanta Petrarca identifica Valchiusa come il porto in cui a stento trova ricovero la «sconquassata navicella della mia vita» [in hunc portum conquassatam vite naviculam] (Fam. XV 8), lontano dall'ambiente infernale della vicina Avignone, da cui emana, come da una nefasta Babilonia, un puzzo ripugnante che giunge a inquinare anche quel lembo di paradiso terrestre. ${ }^{17}$ Questa vicinanza con la limitrofa città papale, dalla quale giungono i miasmi che inquinano la natura del suo luogo prediletto e soprattutto l'eco delle cure mondane e curiali che confondono la sua mente

[Hec tibi quoque, Sorgie ad fontem, quo rursus e tanto Italie naufragio velut in portum fugi, et preteriti dolens et venturi trepidus, dictabam Kalendis Sextilibus, intempesta nocte.], Francesco Petrarca, Opere, cit.

15. «quo rursus e tanto Italie naufragio velut in portum fugi, et preterit dolens et venturi trepidus, dictabam Kalendis Sextilibus, intempesta nocte».

16. «Non di Valchiusa luogo alcuno è al mondo / A me più grato ed a’ miei studi adatto. / Vissi in Valchiusa la puerizia, e giovane / A lei tornato, nel suo seno aprico / L'amena valle mi nutrì. Uom fatto / Dolcemente a Valchiusa i migliori anni / Trassi e i candidi stami della vita. / Vecchio, in Valchiusa l'età mia finire, / In Valchiusa incontrar la morte voglio.», Francesco PeTrarca, Opere, cit.

17. Fam. XV 8, 5: «Poiché, anche dentro al porto mi guardo attorno con timore, e soprattutto mi spaventa la vicina Babilonia, che chiamano Curia roman; nome strano, che nulla è meno romano, nulla è ivi più in odio di Roma; e la sua vicinanza, la sua vista, il suo fetore è veramente terribile e nemico d'ogni felicità, tanto che basterebbe da solo a mettermi in fuga, per non parlare dei resti delle passate tempeste, che molto da vicino perseguitavano fino in questo porto la sconquassata navicella della mia vita» [In ipso igitur portu timens multa circumspicio, sed ante omnia vicina Babilone deterreor, quam romanam curiam dicunt; mirum, cum nichil minus quam romana, nichil illi usquam invisius Roma sit. Huius certe vicinitas ac prospectus odorque terribilis et felicitati nimis infestus est; ille me vel solus hinc pelleret, ut omittam preteritarum reliquias tempestatum, que de tam propinquo usque in hunc portum conquassatam vite naviculam persequuntur], Francesco Petrarca, Opere, cit. 
solitaria, insieme alla nostalgia sempre crescente per l'Italia, dovettero essere le cause che lo spinsero ad abbandonare per sempre la sorgente delle dolci acque da cui erano scaturite così grandi opere poetiche, filosofiche e morali. ${ }^{18}$

Ma prima di giungere al pessimismo globale della vecchiaia, quello espresso nella lettera a Guido Sette in cui sembra non ci sia più spazio per le illusioni, Petrarca rifacendosi alla sua esperienza personale e quindi alla contrapposizione Avignone-Valchiusa, tende a leggere la realtà alla luce della contrapposizione città-campagna, caricando una di valori assolutamente negativi quanto l'altra di positivi, apparentemente semplificando una questione che egli stesso pone come più complessa. Se infatti partiamo dalle sue valutazioni, alcune addirittura molto positive, di Roma, Venezia e Napoli ci rendiamo conto che per lui ogni grande città, per il fatto di essere un centro di agglomerazione di masse, è di per sé un ostacolo alla crescita della vita interiore, ma che anche alcune città di dimensioni ridotte superano abbondantemente le grandi per viltà e nefandezza. È ovvio che la palma della perfidia spetta ad Avignone, sede di una curia corrotta e viziosa. Ad essa dedica, tra l'altro, i violentissimi sonetti antibabilonici ( $R V F$ 136, 137, 138): «Nido di tradimento, in cui si cova / quanto mal per lo mondo oggi si spande» (136: 5-6); «Fontana di dolore, albergo d'ira, / scola di errori e tempio d'eresia» (138: 1-2). E anche se Petrarca, nella sua visione politica, contrappone il Tevere al fiume Durance e Roma a Avignone-Babilonia (come dice in Sine nomine 18), ${ }^{19}$ nella

18. Fam. XI, 4-5, 8-9: «Due cose mi soltanto mi dispiacciono, che è lontana dall'Italia, verso la quale l'animo mio è naturalmente attratto e troppo vicina a questa Babilonia d'occidente, la peggiore di tutte e simile all'inferno, cui fugge e abborre la mia natura.[...] Quando a tutti avrò dato l'ultimo addio, fuggirò "quella terra crudele e il lido avaro", e in quel mio rifugio, che si nasconde a quindici miglia di là, presso la sonora sorgente della Sorga, tra fiumi e selve e tra i vari libri, che da quattr'anni silenziosi e chiusi da rustico custode guardati mi aspettavano, passerò in quiete solitaria il resto dell'estate [...]. In autunno farò ritorno, spero, insieme coi libri della mia biblioteca che ho stabilito di portare in Italia.» [Duo ibi sunt fateor adversa animo, et quod ab Italia locus abest, ad quam me naturalis motus attrahit, et quod vicina nimis est Babilon hec occidentalis, rerum pessima Ereboque simillima, unde me natura itidem dehortatur ac retrahit mea. [...] cumque omnibus supremum vale dixero, «crudeles terras» et "avarum» vere «litus» effugere atque in prefato rure nostro, quod procul inde quindecim passuum milibus ad preclarum ac sonorum Sorgie fontem latet, inter silvas ac flumina interque libellos varios, qui ibidem sub rustico custode vincti et taciti iam me quadriennium expectarunt, quod superfuerit estatis solitaria quiete transigere, [9] ne si mox vestigia nota relegero, corpusculum hoc, continuis licet ab infantia laboribus exercitum, nimio forsan ardore conficiam, cui parco non ob aliud nisi quo diutius torqueam ac fatigem. [...] Autumnus me revehet, ut spero, simul et libellos ipsos bibliothece itale quos inferre disposui.], Francesco Petrarca, Opere, cit.

19. Cfr. Ugo DotTI, Petrarca civile. Alle origine dell'itellettuale moderno, Roma: Donzelli, 2001, p. 149, ma anche p. 128: «In Avignone — noi sappiamo- egli vedeva simbolicamente la corruzione della Chiesa di Cristo e l'intollerabile esilio di Pietro; da un certo punto in poi sappiamo anche che nei confronti di essa userà quasi costantemente un'espressione non in sé nuova ma fortunata: la "Babilonia infernale" "; Ugo DOTTI, Petrarca e la scoperta della coscienza moderna, Milano: Feltrinelli, 1978, p. 95: «il suo distacco da Avignone e dall'ambiente della Curia, se ancora non può considerarsi definitivo [nel 1351], può tuttavia ritenersi, al di là dei rapporti formali, assai profondo. Un distacco, anzitutto, dal protettore Giovanni 
sua visione personale, intellettuale e umana l'autentico opposto al labirinto della curia avignonese è il silenzio di Valchiusa, visto che vi soggiornò durante circa dieci anni, suddivisi in quattro periodi diversi: 1337-1351, 1342-1343, $1345-1347$ e $1351-1353$.

Nel 1337, di ritorno da Roma, si trasferì a Valchiusa nella casa che aveva appena acquistato nelle vicinanze della sorgente della Sorga. Questo rifugio di campagna costituì da allora l'esempio più paradigmatico dello stile di vita semplice e ritirato che aveva scelto per sé e che alternava a lunghi viaggi e soggiorni, anch'essi di una certa durata, in città o dimore signorili. Questo luogo, al quale se ne aggiungeranno altri come l'altipiano di Selvapiana, Arquà o la Certosa di Garegnano, sarà sempre il suo favorito e l'unico nel quale realizzerà l'autentica esperienza dell'importanza e del valore della vita solitaria, da lui elogiata in molti passi della sua opera e non solo nel De vita solitaria, per quanto questa sia dedicata interamente alla libertà dell'uomo solitario in opposizione alla vita del cittadino.

Qui nel posto che è diventato paesaggio, cioè sfondo alla sua attività creativa, Petrarca racconta di aver sentito l'impulso della creazione letteraria più che in qualsiasi altro luogo:

È li, e mi piace ricordarlo che cominciai a scrivere la mia Africa con tanto impeto e dedizione che ora, mentre sto adoperando la lima, provo in qualche modo paura per la mia adudacia e la grandiosità dell'opera; è lì che ho composto una non piccola parte delle lettere in prosa ed in versi e quasi tutto il Bucolicum carmen, e in un tempo così breve che, se lo sapessi, ne stupiresti. Non c'è infatti luogo che mi abbia dato più riposo o fornito stimoli più efficaci. Fu proprio quella solitudine a darmi il coraggio di raccogliere in un solo libro gli uomini illustri di tutti i luoghi e di tutti i tempi; a spingermi a lodare in singoli libri le singolari bellezze della vita solitaria e della pace dei chiostri;a farmi infine sperare di poter lenire tra la frescura di quelle ombre l'ardore giovanile che, come sai, mi infiammò. [...] Senonché, bruciato da quegli stessi ardori che portavo con me e privo in tanta solitudine di chi mi aiutasse contro quel fuoco, arsi così disperatamente. E così, erompendo dalla mia bocca, la fiamma del cuore riempiva il cielo e la valle di un mormorìo infelice ma, come ad alcuni parve, anche dolce, e da ciò nacquero quelle rime in volgare dei miei giovanili tormenti dei quali oggi mi vergogno e mi pento ma che pure sembrano molto gradite, como possiamo vedere, a coloro che sono colpiti dallo stesso male. $\mathrm{Ma}$ a che scopo parlar tanto? Se si confrontasse tutto ciò che ho scritto altrove con ciò che ho scritto a Valchiusa, questo luogo, a mio giudizio, supererebbe qualsiasi altro (Fam. VIII 3, 11 sgg.). ${ }^{20}$

Colonna, al cui servizio il giovane Francesco era entrato nel 1330, e che ci è testimoniato, nei suoi aspetti anche umani, dall'egloga VIII, Divortium. Scritta durante l'estate del 1347, essa documenta molto da vicino non soltanto la crisi dei rapporti tra Petrarca e il Colonna, ma la più vasta crisi in cui sembra sia stato posto lo scrittore dopo gli avvenimenti romani di Cola».

20. "Illic — “iuvat" enim "meminisse”- Africam meam cepi, tanto impetu tantoque nisu animi, ut nunc limam per eadem referens vestigia, ipse meam audaciam et magna operis fundamenta quodammodo perhorrescam; illic et epystolarum utriusque stili partem non exi- 
Nel 1353 si conclude definitivamente la sua permanenza nell'Elicona transalpino. Essa ha lasciato una traccia indelebile nel suo spirito di poeta e sul piano letterario una gran quantità di opere quivi avviate o portate a termine, nel ricco e vivace spazio di solitudine da lui creatosi, animato soltanto dalla presenza dei classici. Un anno prima scrive al suo amico Jacopo da Firenze una lettera — che sarà in seguito ritenuta il primo manifesto dell'Umanesimodai toni per così dire quasi allucinati, in cui racconta che durante il suo ritiro nel Parnaso provenzale ha ricevuto la visita di Cicerone il quale, impressionato dal luogo, dopo dieci giorni di convivenza gli confessa che la fonte di Valchiusa è superiore alla Ninfa campana e alla siciliana Aretusa. Lo stesso argomento è trattato in un'altra del 1353, indirizzata a Zanobi da Strada, in cui dice:

Ecco la mia vita: mi alzo a mezza notte, di primo mattino esco di casa, ma così nei campi come in casa studio, leggo, scrivo, tengo quanto posso lontano dai miei occhi il sonno, dal corpo la mollezza, dall'anima i piaceri, dagli affari la pigrizia. Ogni giorno mi aggiro tra aridi monti, rugiadose valli e spelonche, passeggio spesso sull'una e l'altra riva della Sorga, senza incontrare alcuno che strepiti, mi accompagni, mi guidi, se non le mie cure, che ogni giorno si fanno meno acute e moleste; e ora cacciandole davante a me, ora lasciandole addietro, memore del passato delibero sull'avvenire. ${ }^{21}$

E conclude dicendo che, fin tanto che la ruota della fortuna non cambierà direzione (cosa che sta effettivamente accadendo, visto che proprio in quest'anno lascerà quel luogo per sempre):

guam et pene totum Bucolicum carmen absolvi, quam brevi dierum spatio si noris, stupeas. [12] Nullus locus aut plus otii prebuit aut stimulos acriores: ex omnibus terris ac seculis illustres viros in unum contrahendi illa michi solitudo dedit animum; solitariam vitam religiosumque otium singula ibi singulis voluminibus perstringenda et laudanda suscepi; denique, iuvenilem estum qui me multos annos torruit, ut nosti, sperans illis umbraculis lenire, eo iam inde ab adolescentia sepe confugere velut in arcem munitissimam solebam. [13] Sed, heu michi incauto! ipsa nempe remedia in exitium vertebantur; nam et his quas mecum advexeram, curis incendentibus et in tanta solitudine nullo prorsus ad incendium accurrente, desperatius urebar. Itaque per os meum flamma cordis erumpens, miserabili, sed ut quidam dixerunt, dulci murmure valles celumque complebat; hinc illa vulgaria iuvenilium laborum meorum cantica, quorum hodie pudet ac penitet, sed eodem morbo affectis, ut videmus, acceptissima. [14] Quid multa? si quecunque alibi cum his que ibi scripsi conferantur, loca omnia locus ille, me iudice, hactenus superet», Francesco PeTrarca, Opere, cit. Per il mito di Valchiusa nella storia politico-letteraria da Petrarca in poi, si veda Eve DuPERRAY, Lor des mots. Une lecture de Pétrarque et du mythe littéraire de Vaucluse des origines à l'orée du XXe siècle. Histoire du pétrarquisme en France, Paris: Publications de la Sorbonne, 1997.

21. «Hec vita mea est: media nocte consurgo, primo mane domo egredior, sed non aliter in campis quam domi studeo cogito lego scribo, somnum quantum fieri potest ab oculis meis arceo, a corpore mollitiem, ab animo voluptates, ab operatione torporem. [11] Totis diebus aridos montes, roscidas valles atque antra circumeo, utranque Sorgie ripam sepe remetior, nullo qui obstrepat obvio, nullo comite, nullo duce, nisi curis meis minus in dies acribus ac molestis. Illas ante retroque transmittens, preteritorum memor ventura delibero.» (Fam. XV 3), Francesco Petrarca, Opere, cit. 
qui ho posto la mia Roma, la mia Atene, la mia patria; qui tutti gli amici che ho e che ebbi, e non solo quelli provati per familiare consorzio e vissuti con me, ma quelli che vissero or son molti secoli, ch'io conosco soltanto per il tramite delle lettere e ammiro per le loro imprese, per l'indole, i costumi, la vita, la lingua o l'ingegno, qui, venuti in questa stretta valle da ogni luogo e da ogni età, io spesso raccolgo, e più dolcemente con essi parlo che con quelli che credono di esser vivi, perché quando nel respirare emettono il loro fiato pestifero, ne vedono la nebbia nell'aria gelata. Così libero e sicuro vado vagando, e tra tali compagni son solo; sto dove voglio, più che posso sto con me stesso; spesso anche con te e con quell'uomo grande e ottimo, che sebbene non abbia mai visto - mirabile a dire - vedo a ogni momento, col quale ti è dato conversare, il mio nome non invecchi. ${ }^{22}$

E a conferma di ciò, in quegli stessi anni rappresenta se stesso per mezzo di un rebus i cui elementi raffigurano la sorgente della Sorga. Petrarca è essenzialmente una sorgente.

\section{Il rebus del manoscritto della Historia Naturalis di Plinio}

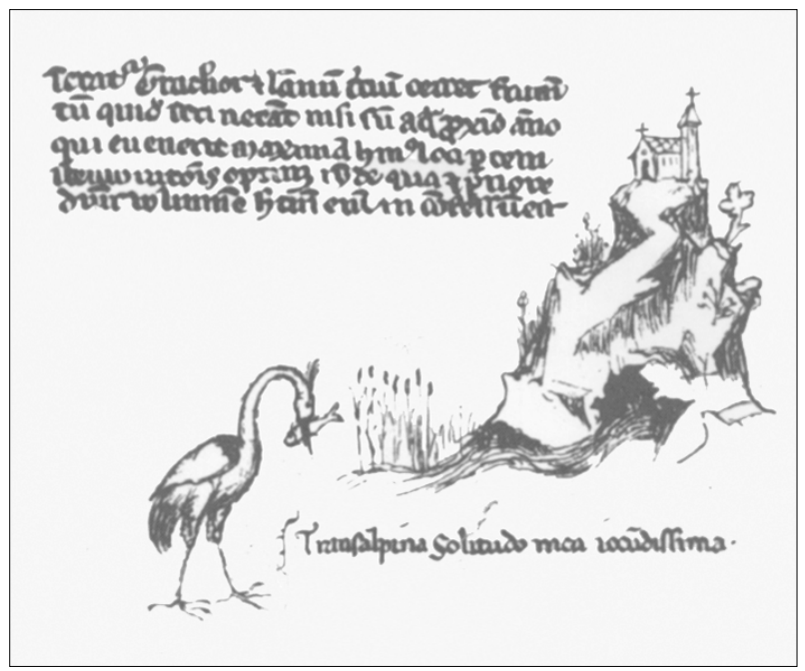

22. "Interea equidem hic michi Romam, hic Athenas, hic patriam ipsam mente constituo; hic omnes quos habeo amicos vel quos habui, nec tantum familiari convictu probatos et qui mecum vixerunt, sed qui multis ante me seculis obierunt, solo michi cognitos benificio literarum, quorum sive res gestas atque animum sive mores vitamque sive linguam et ingenium miror, ex omnibus locis atque omni evo in hanc exiguam vallem sepe contraho cupidiusque cum illis versor quam cum his qui sibi vivere videntur, quotiens rancidum nescio quid spirantes, gelido in aere sui halitus videre vestigium. [15] Sic liber ac securus vagor et talibus comitibus solus sum; ubi volo sum; quotiens possum mecum sum; sepe etiam tecum et cum illo viro optimo maximo, quem cum nunquam viderim, — dictu mirum - omnibus horis video, apud quem, queso, nomen meum dum tu secum loqui poteris, non senescat.» (Fam. XV 3), Francesco PeTrarCA, Opere, cit. 
Il 6 luglio 1350 Petrarca acquista a Mantova un codice della Naturalis historia di Plinio il Vecchio (oggi in Bibl. vat. lat. 6802, f. 143 v), che riempirà di interessanti annotazioni geografiche, morali filosofiche e di storia dell'arte. In calce al foglio (XVIII, 51, 90) nel quale Plinio parla del fiume Sorga ( Est in Narbonensi provincia nobilis fons Orgae nomine. In eo herbae nascuntur»; Petrarca corrigió "fons Orgae» por «fons Sorgie») c'è un disegno, a quanto pare autografo, sotto si legge un'epigrafe latina: «Transalpina solitudo mea iocundissima». In esso è raffigurata una rupe con in cima una chiesa a cui si accede per un sentiero ampio e ben tracciato che passa attraverso precipizi coperti di alberi e arbusti. Ai piedi di questa grande rupe c'è un'immensa e oscura voragine da cui sgorga la sorgente nel cui alveo fioriscono giunchi e stiance. Sulla riva un volatile dalle lunghe zampe (un airone, un pellicano, una cicogna o una gru) sta ingoiando il pesce che la appena catturato.

Il paesaggio, tracciato con stilizzata sobrietà, è costituito dunque dalla chiesa in cima alla rocca, dalla rocca stessa, dall'ampia e oscura sorgente da cui sgorga il rivo ondulato e infine dall'uccello. Secondo un'ottica di osservatori moderni, si potrebbe addirittura ritenerlo uno schizzo quasi realistico dell'amata Valchiusa, il suo Parnaso transalpino. Ma ci inganneremmo. Si tratta invece di un paesaggio della memoria, della nostalgia e del desiderio. Realizzato molto probabilmente a Mantova a tre anni dal suo ultimo, anche se non ancora definitivo, abbandono delle rive della Sorga e un anno prima di farvi ritorno, lo schizzo fonde in sé questi tre movimenti dello spirito. C'è chi ha ipotizzato che sia stato realizzato sotto la guida dell'amico Giovanni Boccaccio, «dilettante esperto di disegno", o addirittura eseguito a quattro mani. ${ }^{23}$ E molto probabile che in questo disegno sia presente il ricordo ${ }^{24}$ della miniatura del Maestro del Codice di San Giorgio nel codice Stefaneschi dell'Archivio di S. Pietro, in cui appare, da destra a sinistra, un monte coronato da un castello dal quale si affaccia una moltitudine di persone intente a seguire la lotta che il cavaliere San Giorgio ha ingaggiato contro il dragone nella pianura sottostante punteggiata da piante lacustri. È fácilmente ipotizzabile che Petrarca abbia conosciuto il codice Stefaneschi (dell'Archivio di S. Pietro, Ms. C. 129), dato che l'artista, alunno di Simone Martini, ${ }^{25}$ era molto conosciuto nella curia avi-

23. Vittore BRANCA, "Interstestualità fra Petrarca e Boccaccio», in Lectura Petrarce XIV, 1994.

24. Cfr. Karlheinz Stierle, Francesco Petrarca. Ein Intellektueller im Europa des 14. Jahrhunders, Munchen-Wien: Carl Hanser Verlag, p. 64-65; Bernhard DEGENHART - Annegrit SCHMITT, Corpus der italianischen Zeichnungen, 1300-1450, Berlin: Mann, 1980, p. 131: «Das Gewässer mit Schilf und Reiher bei dem eng mit Simone zusammenhängenden Georgsmeister (Abb. 136) vereint dieselben Elementen wie Petrarcas Zeichnung auf fol. 143 verso.»; Giovanni Romano, Studi sul paesaggio, Torino: Einaudi, 1978, p. 29.

25. Bernhard DEgENHARDT- Annegrit SCHMITT, Op. cit., p. 87: «Eine änhliche Wechselwirkung aus sienesischen und französichen Elementen zeigt der Maestro del Codice di San Giorgio, sowohl in der verschieden datierten, aber ünerreinstimmend in die erste Trecento hälfte versetzten Stefaneschi-Manuskript des Archivo di S. Pietro (Ms. C. 129), als auch in der Bibel in Boulogne s. M. (Ms. 86)»; cfr. anche Maria POGETTO, Il Maestro del Codice di San Giorgio e il Cardinale Jacopo Stefaneschi, Firenze: Edam, 1981. 
gnonese. ${ }^{26} \mathrm{Ma}$ oltre alle coincidenze nella struttura e nella disposizione degli elementi, bisogna considerare anche le differenze tematiche, giacché il poeta, a differenza dell'autore di San Giorgio, non ha in mente una scena epico-allegorica, quanto un'autorappresentazione sotto forma di rebus nella quale apparentemente non c'è nessun elemento umano, ma soltanto dei simboli.

Ciò nonostante è degno di rilievo il fatto che lo sguardo di Petrarca si sia soffermato proprio sull'immagine di San Giorgio che lotta con il drago; anche se nel rebus petrarchesco il cavaliere e il drago sono stati sostituiti da uno dei trampolieri che nella miniatura del maestro costituivano soltanto un mero dettaglio decorativo. Ė probabile dunque che sotto la calma del rebus del poeta ci sia una visione epico-eroica dell'esistenza umana che consiste nel trasformare la "pietra morta" in "pietra viva", come scrive Corrado Bologna in un suo acutissimo articolo:

Per realizzare questa metamorfosi culturale della natura selvaggi Francesco ingaggiò una guerra armata («bellum [...] ingens», "certamen», «arma») con le Ninfe della fonte sgorganti $[\ldots]$ una guerra fondatrice di nuove dimensioni del reale. ${ }^{27}$

Si è giunti anche a mettere in relazione il disegno di Petrarca con quello che l'amico Simone Martini dedicò a Virgilio sul frontespizio di un codice miniato contenente alcune opere con il commento di Servio (Milano, Biblioteca ambrosiana, ms. S.P. 10,27, Virgilius cum notis Petrarcae, 1340 c.) [Apéndice I B]. Questo accostamento, tuttavia, non viene suggerito dalla somiglianza delle due composizioni, quanto piuttosto dalla comune intenzione didatticoallegorica. Infatti il pittore senese, residente ad Avignone e grande amico del poeta che di lui possiede alcuni quadri, tra i quali, amatissimo, il ritratto di Laura del quale fa un elogio senza uguali:

E sol ad una imagine m'attegno, che fe' non Zeusi o Prasitele o Fidia, ma miglior maestro e di più alto ingegno (RVF 130)

unisce in questo codice il simbolismo didattico e una raffigurazione paesaggistica della natura. Questa miniatura, considerata dagli esperti come una delle

26. Bernhard DegenhardT- Annegrit SChMitT, Op. cit., p. 130-131: «Der liebe und verehrte Freund Simone Martini liess Petrarca sicher Werke —und Zeichnungen! — sehen, die wir nicht mehr kennen».

27. Corrado BolognA, «PetrArca petroso», in Critica del testo, n. VI/1, 2003, p. 366-420: 382-383.

Il graziosissimo racconto della grande guerra per i confini («bellum de finibus ingens») che il poeta ingaggia con le ninfe si trova nell'epistola metrica II 1 . In essa le divinità della foresta sostengono i loro diritti sui quei luoghi contro le pretese del poeta di trasformarli in rifugio alle Muse, mostrando così di preferire «novem anus quod mille puellis». Alla fine il poeta avrà la meglio sulle Ninfe («Pellimus his tota Nimphas regione subactas / erigimusque sacris mansura palatia Musis», v. 52-53). 
opere fondamentali per la ricostruzione dell'attività avignonese dell'artista, rappresenta Servio nell'atto di sollevare il velo delle oscurità di Virgilio, che si trova disteso a destra. A fianco di Servio c'è Enea, mentre il contadino e il pastore in primo piano rappresentano rispettivamente le Bucoliche e le Georgiche; al centro due coppie di esametri su epigrafi commemorano Virgilio, frutto della terra italica, e Servio, capace di svelare i suoi segreti.

Da queste due miniature bisogna partire per sottolineare le differenze e, conseguentemente, la novità introdotta dalla visione petrarchista del paesaggio, sia rispetto ad altri artisti sia in relazione con altre forme di rappresentazioni, anche più stilizzate e allegoriche.

$\grave{E}$ difficile non interpretare questo disegno come un emblema personale del poeta aretino che in esso riassume la sua visione idealizzata di un luogo concreto e, allo stesso tempo, la sua precisa collocazione in questo stesso luogo e nel mondo in generale (o forse il suo desiderio di stabilirvisi). A maggior ragione, poi, se questo disegno coincide pienamente con molte sue descrizioni di Valchiusa o di Selvapiana, così come leggiamo in Epyst. metr. I 6:

\author{
Stat colle virenti \\ Silva ingens, Planeque tenet licet ardua nomen. \\ Hic solem procul aerias avertere fagos \\ ac teneras variare solum concorditer herbas \\ mensibus estivis videas; hic brachia Cancri \\ temperat unda recens, atque ora iubamque Leonis \\ dulces vicinis feriunt ex montibus aure. [...] \\ Mille nemus volucrum species ac mille ferarum \\ circumeunt habitantque sacrum gelidusque per umbram \\ fons ruit; irriguo pubescunt gramina flexu. \\ Floreus in medio torus est, quem cespite nullo \\ erexit manus artificis, sed amica poetis \\ ipsa suis natura locum mediata creavit. [...] \\ Intus odor mirus statiosque simillima Campis \\ Elysiis profugisque domus placidissima Musis. \\ Defereor huc solus furtim sociosque fefelli. \\ (vv. 23-29, 33-38, 45-47) 28
}

E tuttavia, quale significato attribuire alle diverse parti che costituiscono la miniatura? Possiamo mettere in relazione l'insieme costituito dalla chiesa, la rupe e la sorgente con il suo nome, inteso come Petra-Arca, arca di pietra?

28. «Sta su un verde colle una gran selva, che sebbene posta in alto è chiamata Piana. Ivi nei mesi estivi tu vedresti aerei faggi protegger dal sole e tenere erbe screziare il suolo; ivi una fonte sorgiva tempera il calore delle branche del Cancro, e dai vicini monti dolci aure colpiscono la criniera e la testa del Leone. [...] Mille specie di uccelli e di fiere abitano e percorrono il sacro bosco; scorre nell'ombra un fresco rivo e tra i suoi irighi meandri cresce rigogliosa l'erba. In mezzo è un fiorito seggio che nessuno artefice compose in zolle, ma la natura stessa amica ai poeti scelse e costrù in luogo adatto. [...] Dentro aleggia un soave odore; è questo un tranquillo ricovero alle Muse, in tutto simile ai Campi Elisi. La solo e furtivo io mi reco, fuggendo ogni compagnia.», Francesco PetrarCA, Epystole metrice, Roma: Biblioteca Italiana, 2003. 
Secondo quanto ha messo in rilievo in un suo interessantissimo articolo Corrado Bologna, ${ }^{29}$ l'epoca in cui Petrarca decise di stabilirsi a Valchiusa coincide, molto probabilmente, con la sua decisione di «rielaborare e trasformare nel proprio cognome il nome del Padre». Forse fu proprio in questa valle, intorno al 1340, che Petrarca «adottò — come scrive Wilkins - il nome di Petrarca, al posto di quello usato fino a quel momento, vale a dire Petracchi», ${ }^{30}$ non solo perché questa forma gli doveva suonare "più latineggiante, eufonica e nobile», ma perché conteneva gli elementi di una vera e propria mitologia personale, che sarà confermata in seguito dalla miniatura autografa.

La roccia, in tal senso, raffigurerebbe un'arca di pietra dalla cima della quale il cristiano, chiaramente simboleggiato dalla chiesetta, dal campanile e dalle due croci, guarda al mondo, però ben ancorato in un porto sicuro dalle acque colme di vita ("come m' à concio 'l foco / di questa viva pietra, ov' io $\mathrm{m}$ ' appoggio.» $R V F \mathrm{~L}$ 78). A voler andar oltre, nella chiesetta potremmo vedere raffigurato il ponte di comando della mente, il "cassar della mente», di cui parlava Cavalcanti in Vedeste, al mio parere, onne valore, e il cui modello è l'arx mentis della tradizione classica e medievale, ${ }^{31}$ così ben trattato da Bologna nel citato articolo, che si condensa nel nome e nel disegno di Petrarca come allegoria di una nuova sapienza che vuole essere arca di salvezza; di pietra sì, ma di pietra viva, cioè, spirituale. Nell'Epistola metrica (I 6) a Giacomo Colonna, l'autore mette in relazione la roccia, il rifugio sicuro al lato del fiume («in litore saxum, / naufragiis tutumque me is aptumque putavi») con la «rocca del cuore» («cordis ad arcem») difesa dagli eserciti del sangue. Tra la chiesa e la sorgente, così imponente e alla quale il poeta ha dedicato delle parole a dir poco centrali per questo nostro discorso nel DVS II xiv, ${ }^{32}$ si potrebbe tentare di stabilire la stessa relazione dialettica che in

29. Corrado Bologna, op. cit. Cfr. anche Renzo CeSERANI, «Petrarca»: il nome come auto-reinvenzione poetica, in "Quaderni petrarcheschi», IV, 1987, p. 121-37 e Corrado BOLOGNA, "Ascensioni spirituali», in Les montagnes de l'esprit: imaginaire et histoire de la montagne à la Renaissance (ed. R. Gorris Camos), Quart (AO), Musumeci, 2005, p. 19-56.

30. Ernst Hatch Wilkins, La vita del Petrarca, Milano: Feltrinelli, 20032 [Life of Petrarch, Chicago: University of Chicago, 1961).

31. L'arx rationis di Cicerone e di Boezio assediata dalla passione è stata studiata da Roberto CRESPO, «Il "casser de la mente" cavalcantiano e l' "arx mentis" della tradizione mediolatina», Quaderni di Semantica, a. I, n.1, (gen.-giu. 1980), p. 135-141 e Corrado BOlOGNA, «PetrArca petroso», cit.

32. Perché mette in rapporto proprio la tradizione classica delle sorgenti e dei fiumi con quella cristiana: «Qui anche tu puoi essere libero e padrone e solitario, cosa che capita di rado. Potrai tu disprezzare questo tuo luogo, che nei forestieri incute riverenza e stupore? «Una speleonca» dice Seneca "che tenga sospesa una montagna su rocce profondamente corrose, una spelonca scavata in tanta ampiezza per cause non artificiali ma naturali, colpirà il tuo animo di un religioso terrore». Se questo è vero, dove mai trovare, di grazia, una rotta più augusta? [...] E ancora, se sono vere queste parole: «Dove un gran fiume sbocca improvviso da un luogo nascosto ci sono degli altari», dove, dico, potrebbero più degnamente esserci degli altari? E altari io penso già da tempo d'innalzare [...] là, nel mio giardinetto che sovrasta le sorgenti e giace ai piedi delle rocce: non alle Ninfe, come Seneca diceva, né alle divinità delle sorgenti e dei fiumi, ma a Maria, di cui il parto ineffabile e la feconda verginità rovesciò tutte le are e i templi degli déi. Le stessa forse mi assiterà, affinché possa io una buona volta condurre a termine ciò che da molto tempo e, se non erro, santamente desidero.» 
Petrarca troviamo tra cristianesimo e paganesimo: il cristianesimo costituisce la nave ben ancorata fluttuante sulle vive acque della mitologia pagana e della stessa Bibbia, in un sincretismo iconografico per cui le due parti si alimentano reciprocamente. La fonte delle Muse che Pegaso, il mitico cavallo alato, fa scaturire dalla roccia con un colpo di zoccolo, si innesta nella tradizione biblica dell'acqua viva (ú o o $\varsigma \omega \gamma \gamma$ ), là dove si parla di Dio come sorgente di vita, dello Spirito Santo e del rito del Battesimo. ${ }^{33}$ In Ezechiele e in Zaccaria quest'acqua viva è l'emanazione profetica della vita di Dio: «Una acqua viva sgorgherà da Gerusalemme» (Zaccaria, XIV, 8). Ezechiele (47, 1-12), da parte sua, parla di un angelo che lo conduce ad un tempio sotto il quale l'acqua scorre verso oriente e che gli rivela che quelle acque possono sanare ogni male:

Ogni essere vivente che si muove dovunque arriva il fiume, vivrà: il pesce vi sarà abbondantissimo, perché quelle acque dove giungono, risanano e là dove giungerà il torrente tutto rivivrà. [...] Lungo il fiume, su una riva e sull'altra, crescerà ogni sorta di alberi da frutto, le cui fronde non appassiranno: i loro frutti non cesseranno e ogni mese matureranno, perché le loro acque sgorgano dal santuario.

E ancora in Giovanni 4: 13-14: "Gesù gli rispose: "Chi beve quest'acqua avrà sete di nuovo, ma chi berrà l'acqua che gli darò io, non avrà mai più sete. Anzi, l'acqua che gli darò, diventerà in lui una sorgente da cui sgorga vita eterna”». La roccia assume le connotazioni dell'acqua. L'acqua è viva. L'acqua scaturisce dalla roccia. Dunque la roccia, la pietra, è viva.

Criptogramma e rebus si scambiano a vicenda argomenti per edificare e riaffermare il mito personale intorno al quale nasce il nuovo poeta, l'umanista con la mente cristiana e la base pagana; puro progetto, desiderio di presentarsi come pietra solida, tetragona, inamovibile, nel cui interno, tuttavia, scorrono le acque fresche e correnti dei sentimenti: il pensiero del cuore.

Vale la pena ricordare qui che il mito della solitudine va di pari passo con quello dell'elevazione di Roma a città più bella del pianeta ("Roma sola mirabilis toto orbe terrarum»), come si può leggere nel foglio 266 v. di questo stessso manoscritto contenente la Historia naturalis di Plinio, nel quale un disegno, che sembra pure autografo, raffigura una chiesa gotica tra mure merlate. Si

[Hic tu quoque, quod rarum, et liber et dominus et presul et solitarius esse potes. Poteris ne tuum locum spernere, qui advenis et reverentiam inicit et stuporem? «Siquis specus» inquit Seneca "saxis penitus exesis montem suspenderit, non manufactis sed naturalibus causis in tantam laxitatem excavatus, animum tuum quadam religionis suspicione percutiet». Si hoc verum est, ubi queso religiosior usquam specus? [...]. Rursus si tertium est verum: "Subita" inquit "ex abdito vasti amnis eruptio aras habet», ubi, oro, dignius are fuerint? Quas ego iampridem [...] illic in ortulo meo, qui fontibus imminet ac rupibus subiacet, erigere meditor, non Nimphis, ut Seneca sentiebat, neque ullis fontium fluminumque numinibus, sed Marie, cuius partus ineffabilis et fecunda virginitas omnes deorum aras ac templa subvertit. Aderit ipsa fortassis, ut quod diu iam et, nisi fallor, pie cupio aliquando perficiam].

33. Così in Geremia, II, 13: "Hanno abbandonato me, la sorgente di acqua viva». Il Cantico dei Cantici parla del «pozzo di acqua viva». 
tratta di un'immagine stilizzata e condensata della idea della città eterna, latina e cristiana che illustra il testo nel quale lo scrittore latino parla della Roma di Caligula e Claudio e per mezzo del quale si fa palese la diversa concezione della citta che Petrarca aveva rispetto a Plinio. ${ }^{34}$

Ma per riprendere l'indagine sul primo rebus autografo di questo manoscritto, c'è da considerare un'altra miniatura del secolo XVI che può suffragarla [Appendice II]. Si tratta del "Mont de sagesse» (Carpentras - BM- ms. 0412, f. 020v) del libro di J. Lemaire de Belges, La concorde des deux langages, rappresentato da una rocca altissima con un castello sulla cima (arx mentis), ai piedi della quale scorre un rivo di acqua fresca. Sul lato destro un monaco indica a un pastore un' iscrizione sulla roccia in cui si legge: «ICI EST LE HAULT ROCH QUI LES NUES SURPASSE DE PLUS HAULT DU MONDE». È evidente che l'autore, chiunque fosse, si è appropriato del sogno pittografico e mitografico con il quale Petrarca aveva inaugurato il mondo moderno, costruendo, alimentando ed esprimendo con quelle rocce e con quelle le acque il suo desiderio d'individuo e il suo spirito fieramente umano, troppo umano.

Se la montagna è l'autorappresentazione del cognome che è seguita alla trasformazione di un nome comune in nome proprio, che cosa rimane da dire circa il secondo elemento, tanto più significativo, del disegno autografo? E plausibile che l'autore abbia voluto rappresentare se stesso come una specie di trampoliere con un pesce nel becco, un volatile, insomma, il cui corpo forma una $f$, segno che egli stesso ripete proprio prima dell'inizio dell' iscrizione latina. Questa potrebbe essere la soluzione del rebus propostoci dal poeta: $f$ (rancesco) Petr-Arca. A suffragare l'interpretazione dell'airone come pittograma di Franciscus, concorrono i diversi luoghi dell'epistole in cui egli fa riferimento alla sua passione per la pesca. Ricordiamo, fra le altre, la Fam. XIII 8 9: «mi piacciono i pesciolini, dei quali il fiume abbonda, e mi diverto soprattutto a pigliarli, maneggiando ami e reti» [quibus hic fluvius abundat, pisciculis delector, nunquam magis quam dum capiuntur, quod studiose etiam inspicio, iuvatque iam hamus et retia tractare $]^{35} \mathrm{e}$, soprattutto, i versi finali dell' Epistola metrica III 4 a Giovanni Colonna:

Retia nunc sunt arma michi cessisse vicissim et cecidisse minas compressaque bella videbis.

Pro gladiis curvos hamos, fallacibus escis

Implicitos, tremulasque sudes, parvumque tridentem,

Piscator modo factus ago, quo terga natantum

34. Cfr. Bernhard DegenhardT- Annegrit Schmitt, Op. cit., p. 130: «schematischen Architekturphantasie auf fol. 266 verso, bei der eine Verbindung mit Rom kaum herzustellen ist») y, sobre todo, Karlheinz STIERLE, Francesco Petrarca. Ein Intellektueller im Europa des 14. Jahrbunders, cit., p. 67-68 («die Zeichnung des neuen Rom aber ganz offensichtlich nicht entspricht. Wieder bringt Petrarcas Kommentar so die Differenz zwischen dem Erfahrungshorizont des Plinius und einem neuzetlichen Erfahrungshorizont zu Bewusstsein».

35. Fam V 14, in cui, affrontando l'argomento del vivere in compagnia di cani, dichiara di poter «essere pescatore, ma non cacciatore, specialmente con cani di quel genere». 
Sistere iam didici duroque affigere saxo.
Primitias en fluminee transmittimus artis.
Et versus quod Clausa domos habet artaque Vallis
Que tibi pisciculos et rustica carmina pascit. ${ }^{36}$

in cui la minuziosa descrizione dell'arte pescatoria ha il suo corrispondente iconografico nell'uccello pescatore del rebus, la cui elegante figura è l'iniziale del nome del poeta mentre il pesce che racchiude nel becco è un chiaro riferimento alla sua passione: $\mathrm{f}$ (ranciscus piscator). Risolto il primo livello ermeneutico, resta da identificare correttamente la tipologia del volatile e il suo eventuale valore simbolico. Per far questo lo confronteremo con diverse altre immagini simili contenute nei bestiari più conosciuti dell'epoca e nei capilettera di diversi manoscritti coevi, senza perdere di vista la possibilità che Petrarca abbia voluto innovare anche in questo terreno.

I bestiari ci concedono un certo margine di libertà circa la corretta interpretazione di questo disegno, nel quale potremmo di volta in volta riconoscere una gru, un airone o una garzetta, una cicogna o addirittura un cigno. Ciascuno di questi pennuti possiede un suo concreto simbolismo, anche se in alcuni casi le loro leggende e significati si intersecano e si confondono. Le gru sogliono essere messe in relazione con il viaggio in gruppo e con il sacrificio dell'individuo per gli altri, poiché una gru rimane vigile mentre le altre dormono e per non addormentarsi a sua volta si mette una pietra sulle zampe, ${ }^{37}$ assurgendo, in tal modo, a simbolo di vigilanza, previsione e prudenza. Nel cristianesimo questo animale simboleggia il fedele, e per questo veniva rappresentato nell'atto di bere da un calice. In un elemento decorativo di ceramica proveniente da Villa Adriana a Tivoli riconosciamo una gru che lotta contro un serpente, a simboleggiare la lotta di Cristo contro il demone. L'analogia stabilita da Dante in Inferno V, 46 tra gru e lussuriosi, lungi da fornirci un dato contrario nella nostra ricerca per una corretta interpretazione dell'uccello raffigurato, potrebbe addirittura fornircene uno che avvalora la tesi dell'autorappresentazione di se stesso come poeta lussurioso, confessione questa contenuta nel Secretum. Questa lettura, tuttavia, ci porterebbe a interpretare il rebus come rappresentazione dell'io contradditorio che appare in molte delle sue pagine autobiografiche e non come raffigurazione delle sue aspirazioni ideali. Le immagini miniate dei pellicani poco si differenziano dalle gru,

36. «Le mie armi sono ora le reti e le nasse insidiose tessute di vimini, che lasciando entrare l'acqua formano un carcere onde ai pesci non è possibile fuggire. Divenuto pescatore, uso per armi gli adunchi ami chiusi dentro fallaci esche e aste flessibili e un piccolo tridente, col quale ho già imparato a colpire il dorso dei pesci e inchiodarli sul duro fondo. Eccoti queste primizie della mia arte fluviale insieme con tanti versi quante sono le case della angusta Valchiusa, che per te serba piccoli pesci e canti agresti», cit. per «Epistole metriche» a cura di E. Bianchi, in Francesco PetrarCA, Rime, trionfi e poesie latine, a cura di F. Neri, G. Martellotti, E. Bianchi e N. Sapegno, Milano-Napoli: Ricciardi, 1951, 776-777.

37. Richard de Fournival, Il bestiario d'amore, a cura di Francesco Zambon, Milano: Pratiche, 1987, p. 58: Cecco D’Ascoli, L'Acerba, p. 96: Cap. XXII: «Hanno le grue ordene e signore, / E quella che conduce spesso grida, / Corregge e amaestra lor tenore». 
dagli aironi e dalle cicogne; e i bestiari mettono in luce la peculiarità propria dei pellicani di ridare la vita ai loro figli dopo aver dato loro la morte. ${ }^{38}$ In questo Zambon vede un parallelismo con il ciclo morte-rinascita della fenice che si getta nel fuoco ogni cinquecento anni per risorgere dalle sue stesse ceneri più bella di prima. ${ }^{39}$ I simboli della cosiddetta canzone delle visioni Standomi un giorno solo a la fenestra ( $R V F$ 323), sono in stretta relazione con quelli del rebus pittografico. La quarta strofa, infatti, descrive una sorgente: "Chiara fontana in quel medesmo bosco / sorgea d'un sasso, et acque fresche e dolci, spargea soavemente mormorando». Queste acque, come Euridice, vengono rapite da un vortice che le trascina nelle viscere della terra. E nella quinta strofa appare «una strania fenice» — simbolo di Laura, ma anche e soprattutto della poesia- «per la selva altera e sola» che sparisce senza quasi lasciar traccia, come del resto tutto in questa poesia, eccetto una profonda malinconia nell'animo del poeta. E la stessa tristezza che ci comunica anche il cigno di $R V F 2360,{ }^{40}$ e che, almeno apparentemente, ha poco a che vedere con la serena idealità del rebus. Per tanto, altre dovranno essere le strade che bisognerà percorrere per decodificare questa raffigurazione. Sulla cicogna leggiamo in De Animalibus che "Avis aquatica est plusquam terrestris, et piscibus pascitur». ${ }^{41}$ Altri bestiari, come $L i$ Bestiares d'amours o Il Libro della natura degli animali (conosciuto anche come Bestiario toscano o, nella redazione veneta, Bestiario tosco-veneto) ci dicono del suo amore coniugale e soprattutto di quello filiale, che in ambito cristologico riportano alla misericordia, alla carità e all'amore filiale nei confronti di Dio. Soltanto pochi bestiari fanno menzione delle note marginali raccolte da Cecco D'Ascoli. ${ }^{42}$ La credenza che questi animali portano i bambini appena nati appesi al becco potrebbe essere sorta, secondo alcuni, dalla relazione che questo uccello ha con le acque creatrici di vita. La cicogna, pescando in esse, trova la vita allo stato embrionale. Comunque di tutte le identificazioni fatte fino-

38. Richard de Fournival, cit., p. 62; Cecco D'Ascoli, cit., p. 87.

39. Santagata stabilisce un rapporto fra la fenice e i pellicano: «A testimonianza del legame sotterraneo che corre tra l'immagine della fenice-pellicano e Valchiusa si osservi che a c. 143v del ms. Par. lat. 6802 contenente Plinio (fonte diretta per la representazione della fenice petrarchesca: cfr. Monti, p. 106-7). Petrarca ha disegnato uno schizzo di Valchiusa che presenta in primo piano un tampoliere con un pesce in bocca, uccello "forse riferibile, vagamente, al pellicano" (Battisti, p. 327)», in Francesco Petrarca, Canzoniere, a cura di Marco Santagata, Milano: Mondadori, 1996, p. 1238-1239.

40. «et già mai poi la mia lingua non tacque / mentre poeteo del suo cader maligno: / ond'io presi col suon color d'un cigno.»

41. Cit. per Francesco ZaMBOn, L'alfabeto simbolico degli animali, Milano: Luni, 2001, p. 192.

42. "Cicogna, quando ha male, el ben conosce, / Chè bene ha forza de l'acqua marina: /Cossì da lei fuggir l'angosce. // Se mai in fallo trova soa compagna / Desdegna e mai con lei non s'avicina, / Sola pensando va per la campagna, // D'animali venenosi si nutrica, / E per lo veneno già mai non l'ffende, / Naturalmente la serpe mastica. [...] // El vizio abbandona desdegnando; / Non teme so veneno, che nel mondo / Occide l'omo; su, nel doce affanno, / Drizza el core verso'l fine e'l bene; / E, soffrendo el corpo el grave pondo, / Vede salute a la gravosa spene. // E posa l'anima con dolcezza e pace / Sopra le stelle, si come a Deo piace.» Per ulteriori informazioni, si veda Alfredo CaTTABIANI, Volario. Simboli, miti e misteri degli esseri alati: uccelli, insetti, creature fantastiche, Milano: Mondadori, 2000, p. 163-169. 
ra, quelle dell'airone (Ardea) o della gazzetta (Egretta) si avvicinano di più all'animale del rebus; anche se non si deve dimenticare che la maggior parte di questi disegni non sono frutto di osservazioni naturali, bensì simboli che possono agglutinare in sé tratti di uccelli diversi. Come tutte queste specie acquatiche, gli uccelli dai lunghi trampoli sono emblema di Cristo, e per estensione, dell'uomo eletto o giusto, come si legge nel De Bestiis et Aliis Rebus, attribuito a Ugo da San Vittore. Il Fisiologo, tuttavia, li associa al diavolo per il fatto di nutrirsi di pesce, simbolo a sua volta di Cristo. ${ }^{43} \mathrm{Nel}$ Medioevo spesso erano raffigurati con nel becco uno zircone [Apéndice IV B ], minerale giallo che, secondo la credenza, induceva alla visione spirituale della realtà. Il trampoliere e la sua pietra simboleggiano, dunque, il raccoglimento interiore e la meditazione necessari per raggiungere la scienza e la saggezza divina: niente di più consono ai desideri e agli ideali del nostro poeta.

Le uniche immagini che contengono l'insieme iconografico dell'uccello e del pesce sono delle miniature che, nella maggior parte, illustrano passi della Bibbia [Apéndice III]. Anche se per mancanza di tempo non ho potuto realizzare un'analisi più dettagliata di queste rappresentazioni, sono convinto che occorre insistere nello studio delle lettere iniziali miniate e in generale delle miniature di volatili trampolieri o comunque acquatici [Apendice IV] per riuscire a interpretare in modo corretto il rebus nel quale Petrarca ha nascosto il suo nome e con il quale ha rappresentato se stesso grazie a queste immagini che ne riassumano tutta la mitopoetica. ${ }^{44}$

\section{La solitudine dell'umanista}

Non tutto è però così rivolto all'interiorità come il rebus e il crittogramma sembrano indicarci. Non tutto è «libro» e «scrittura» per così dire, cioè spazio interiorizzato che gli permette di vivere da qualsiasi parte senza dipendere dalle circostanze esteriori. No. Petrarca tende a creare e a ricreare l'ambiente in cui può pensare e scrivere senza ostacoli innecessari. Per questo già prima di abbandonare definitivamente Valchiusa, il suo Elicona transalpino, costruisce un nuovo Parnaso in terra italiana, a Selvapiana, vicino a Parma. Anche Selvapiana diventerà presto un mito che a sua volta, ${ }^{45}$ già nella vecchiaia, verrà sostituito da Arquà. Tutti scenari e scenografie il più possibile prossimi all' arx mentis o rationis, luoghi terapeutici — porti sicuri- nei quali vale la pena tentare di sanare l'inquietudine profonda e devastante che continuamente lo assale. Una geografia mitica di vita solitaria.

Valchiusa, dunque, non è solo il locus locorum ${ }^{46}$ della vita intellettuale e poetica di Petrarca. È qualcosa di molto più profondo che riguarda l'immagine stes-

43. Alfredo Cattabiani, Op. cit., p. 180.

44. Petrarca, ovviamente, era ben consapevole che il tema del pescatore e del pesce aveva un alto valore simbolico, cfr., tra le altre, le seguenti pagine: XI 6 e XIII 8.

45. Cfr. Fam. V 10 3-4; XII 15 6; XIV 4 9. Cfr. Ugo DotTI, Petrarca a Parma, Roma: Carocci, 2006.

46. Floriana CalitTI, Valchiusa «locus locorum», «Semestrale di Studi (e Testi) italiani», n. 11. 
sa che il poeta vuole dare di sé. Non è solo questione di «essere nel paesaggio», ${ }^{47}$ ma soprattutto «essere paesaggio». E Valchiusa e le varie sue rappresentazioni più o meno stilizzate e idealizzate erano il frutto di questo anelito ad «essere paesaggio». Egli era o desiderava essere veramente quella roccia, quell'uccello, quel paesaggio solitario delimitato da rocce, sorgente, verziere e fauna acuatica, un'oasi fiorita in mezzo a un territorio arido, in pieno deserto. Ai suoi occhi la solitudine terrena appariva colma di valori, un programma dello spirito, un progetto umanista carico di germinanti promesse. L'antica contrapposizione città-campagna diventerà da questo momento, grazie a lui e a Valchiusa, un aspetto imprescindibile nella definizione di un intellettuale e di un artista di valore. La solitudine sarà il topos da cui si salpa (con una pietra-arca che previdentemente e idealmente protegge da naufragi e tempeste) per esplorare e magari conquistare il continente umano in tutti i suoi risvolti, interni ed esterni, individuali e collettivi.

Egli entra in conflitto dialettico con il modello di isolamento monastico perché il suo ritiro è laico, poetico, creativo e solo in ultima analisi anche religioso. $\mathrm{Si}$ oppone in modo aperto e frontale al negotium cittadino e, in primis, alla Curia avignonese che rappresentava la negazione stessa di tutti quegli ideali dei quali era plasmato il nuovo saggio che pretendeva incarnare. Così come si scaglia violentemente contro il modello di intellettuale averroista e aristotelico sulla base della contrapposizione sapere / amare. Una cosa è sapere e un'altra, ben differente, è amare, giacché per lui ogni conoscenza deve essere frutto dell'amore.

Valchiusa, prima di tutto, è la fucina dove ha forgiato la sua concezione del sapere profondamente umano, della soggettività inquieta in contrasto con la conoscenza oggettiva delle cose che tanto criticherà verso la fine della sua vita in un'opera fondamentale per lo studio delle sue idee quale è il De sui ipsius et multorum ignorantia. In opposizione al sapere inutile del magister universitario o, in generale, del dotto scolastico, caratterizzato dalla pratica di un otium inautentico e tutto esteriore ${ }^{48}$ e da un vuoto flatus vocis, Petrarca sottolinea l'esperienza interiore della spiritualità individuale. In questo modo il luogo solitario diviene il locus locorum della vera e unica conoscenza possibile. ${ }^{49}$ È qui dove si afferma la superiorità della coscienza. È qui dove sono forgiati i nuovi mezzi espressivi della soggettività, sia filosofici e morali (epistole, secretum, trattati) sia lirici ( $R V F$, Trionfi, epistole metriche) nei quali riversa i

47. Karlheinz STIERLE, «Paesaggi poetici del Petrarca», in Il paesaggio, Venezia: Marsilio, 1999, p. 121.

48. «E in vero filosofeggiano sulle catedre, ma nella vita fanno i pazzi; danno agli altri precetti, ma sono essi i primi a ignorarli, sono i primi a derogare dalle leggi che hanno imposto e, professatisi alfieri, sono i primi ad abbandonare le file, sono i primi a ribellarsi al impero della virtù» [In cathedris enim philosophantur, in actionibus insaniunt; precipiunt aliis preceptisque suis primi obstant; primi legibus a se datis derogant, et signiferos se professi primi ordines deserunt, primi virtutis imperio rebellant] (DVS II, 12). Cfr. Sonia GENTILI, L'uomo aristotelico alle origini della letteratura italiana, prefazione di Peter Dronke, Roma: Carocci, 2005, p. 218-237.

49. "Il Petrarca non conquistava soltanto la propria indipendenza e autonomia, non si libera soltanto dei fastidi chiassosi della vita cittadina, conquistava finalmente, per così dire, uno degli strumenti necessari alla vera conoscenza», Ugo DOTTI, Petrarca civile, cit. 
diversi farmmenti della sua anima, le molteplici facce della sua interiorità. La verità si può raggiungere solo con gli occhi dell'anima e con le parole del cuore. $^{50}$ La verita che egli ricerca è l'uomo stesso; giace nella natura e nel destino dell'uomo e non delle cose. I luoghi solitari sono il luogo della filosofia, di una filosofia che è cura dell'anima di un uomo malato. Il sapere autentico deve essere qualitativo: è il sapere di un uomo che si dedica alla conoscenza non per accumulare dati ma per migliorare se stesso nel cammino della bontà.

Nel suo caso, inoltre isolamento non significa chiudersi al mondo e fare professione di misantropia, al contrario significa rivolgersi al proprio interno per comprendere meglio se stessi e per capire il mondo e l'umanità. La sua solitudine non è sola, come Petrarca dice in chiusura al De vita solitaria, né il suo ozio è inerte né inutile, anzi è una solitudine che può giovare a molti ["Volo solitudinem non solam, otium non iners nec inutile, sed quod e solitudine prosit multis» (Libro II 14)]. Se non si è in grado di affrontare la vera solitudine degli ermitani, se non si vuole o acora non si può fare a meno di «tutte quelle cose da cui l'animo nostro è preso", bisogna, dunque, crearsi una solitudine amica [«amicam nobis solitudinem») in compagnia dei libri (e dunque dei loro autori con cui colloquiare), dei pochi amici (quasi un duplicato di se stesso) con cui condividere. Aborre la folla, non l'amicizia. La sua è una aristocrazia dello spirito, a la quale, però, non è preclusa una cura per il mondo, anzi, come scrive Dotti: «la solitudine, intesa come luogo ideale di autoeducazione — quasi quell'arrière-boutique di cui dovrà felicemente parlare Michel de Montaigne - si trasforma di fatto in uno stimolo per l'impegno civile e per la conoscenza storica dell'uomo». 51

$\mathrm{Nel}$ panorama dei suoi continui viaggi che lo portano ad andare e venire per tutta Europa, i lunghi periodi di raccoglimento sulle rive della Sorga acquistano il valore di equilibrio tra esperienza del mondo e riflessione sul mondo stesso; e in questi lunghi periodi l'Elicona transalpino costituisce il suo punto fisso - la episteme, per dirla in termini filosofici- 52 sul fluire costante delle sensazioni, sul caos del mondo. Questa è la cifra esatta, il punto nodale per capire pienamente la sua abusata metafora della nave con cui è solito riferirsi

50. «Che se per caso qualcuno disposto a intendere il vero potesse avvicinare l'orecchio del cuore non alla lingua, ma al cuore di uno qualsiasi fra quelli che il volgo credulone guarda stupefatto, credo ammetterà senz'altro d'aver sentido la coscienza confessare con la massima sincerità che la felicità consiste non nella risonanza delle parole, ma nel segreto delle azioni e nell'intimità del vero, non nel plauso che viene dall'esterno o nell'ingannevole stima degli uomini» [Quod si forte datum sit, ut aliquis veri capax non ad linguam sed ad cor horum cuiuspiam, quos credulum vulgus stupet, penitus aurem cordis admoveat, puto sine contentione fatebitur, quod conscientiam nudam ingenue confidentem audierit, felicitatem non verbis sonantibus sed rebus tacitis et veritate intima non externis plausibus aut fallacissima hominum opinione constatare], Francesco PETRARCA, De vita solitaria, cit., Libro I III.

51. Ugo Dотті, Petrarca civile, cit., p. 88.

52. Cfr. SEvErino, Il giogo, p. 27-31 y La filosofia antica, p. 24-25: "lo "stare” (stéme) che si impone "su" (epi) tutto ciò che pretende negare ciò che "sta": lo "stare" che è proprio del sapere innegabile e indubitabile e che per questa innagabilità e indubitabilità si impone "su" ogni avversario che pretenda negarlo o metterlo in dubbio». 
a Valchiusa: la solitudine è la torre d'avvistamento nella quale volontariamente si colloca il nuovo ideale di saggio che non tende alla conoscenza quantitativa, come gli averroisti, ma a quella qualitativa, cioè interiore, secondo gli insegnamenti del suo amato Seneca.

Le metafore dell'immobilità (Arx petrae o saxi) e l'amenità del paesaggio eletto non devono, tuttavia, indurci in errore: né Valchiusa, né qualunque altro luogo solitario possono appagare veramente uno spirito inquieto come il suo, per quanto egli affermi il contrario. Non si tratta di un luogo al riparo completo da temporali e burrasche, giacché sotto questa rocca così solida e compatta continua a scorrere il torrente dell'irrequietezza:

Strano a dirsi, desidero di scrivere, e non so né che cosa né a chi scrivere; e tuttavia - fosca voluttà! - la carta, la penna, l'inchiostro, le notturne veglie mi son più care del sonno e del riposo. Che più? sempre io mi tormento e languisco quando non scrivo; così — nuova stranezza! - se sto in ozio mi stanco, se lavoro mi riposo. Questo mio cuore duro come pietra, che sembra nato dai sassi di Deucalione, quando tutto si volge alla carta, quando ha stancato la mano e gli occhi, allora non soffre più né il freddo né il caldo, allora si sente come avvolto in morbida coltre, e teme di esserne tratto fuori, e si attacca alle membra che si rifiutano di obbedirgli; quando poi per necessità ne è allontanato, allora sente la stanchezza e comincia a prendersi le sue vacanze, come un pigro asinello che si costringa a salire con un gran peso su un monte pieno di sassi, ma presto ricomincia il suo gioco con non minore avidità dell' asinello che torna alla sua piena mangiatoia, come quello del suo pasto tranquillo. Che fare, se non riesco a tralasciar di scrivere né so acconciarmi all'ozio? [...] porto la pena del mio peccato, inquieto in casa senza osar né uscire in pubblico; poiché da ogni parte mi saltano addosso questi frenetici, m’interrogano, mi afferrano, mi danno consigli, disputano, leticano, dicono cose ignote al pastor mantovano e al vecchio meonio. (Fam. XIII 7). ${ }^{53}$

La vera rappresentazione delle figure dell'inquietudine, tuttavia, la ritroveremo soltanto nel Canzoniere, là dove il poeta dispiega l'artificio dell'anima messa a nudo. Ma questo è un altro tema.

53. "Ad Petrum abbatem Sancti Benigni, de eodem deque insanabili scribendi morbo. [1] Mira res dictu, scribere cupio nec quid aut cui scribam scio; et tamen - ferrea voluptas - papirus calamus atramentum nocturneque vigilie somno michi sunt et requie gratiores. Quid multa? crucior semper et langueo nisi dum scribo; sic — nova perplexitas — in quiete laborans in laboribus conquiesco. Durum pectus ac saxeum et quod vere deucalioneis ex lapidibus ortum putes, ubi totum membranis incubuit, ubi digitos oculosque lassavit, tum frigus estumque non sentit, tum sibi compositum lodice mollissima videtur metuitque divelli, et obsequium sibi negantibus membris, heret; [2] ubi vero iubente necessitate distrahitur, tum primum lassari incipit et perinde laborum ferias init, quasi segnis asellus sub iniquo fasce scrupulosum montem iussus ascendere; mox non minus avide ceptum repetit quam plenum fessus ille presepe, nec minus longis lucubrationibus quam is pabulo et quiete reficitur. Quid ergo, quando neque a scribendo cessare neque requiem pati possum? [...][17] ipse criminibus meis plector; nam et domi estuo et vix iam in publicum exire audeo; occurrunt enim omni ex parte frenetici, percontantur arripiunt docent disputant altercantur dicunt que nunquam mantuanus pastor, nunquam meonius senex novit», Francesco PETRARCA, Opere, cit. 


\section{Apendice I}

A. Roma Archivio di San Pietro, Ms C. 129, F. 85.

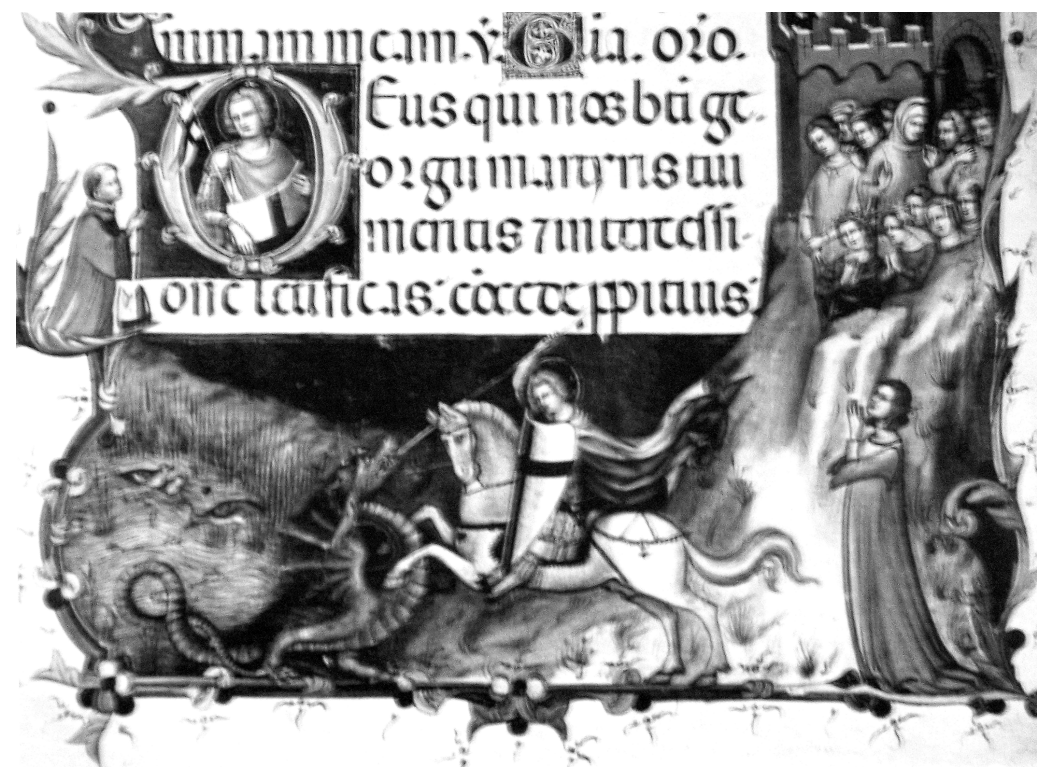

"Maestro del codice di San Giorgio», XIV secolo si chiama l'anonimo artista che illustrò con storie del santo il Codice di S. Giorgio per il cardinale Iacopo Stefaneschi (Roma, Archivio capitolare di S. Pietro). Le miniature, di grande eleganza figurativa e compositiva, rivelano profondi influssi della cultura senese.

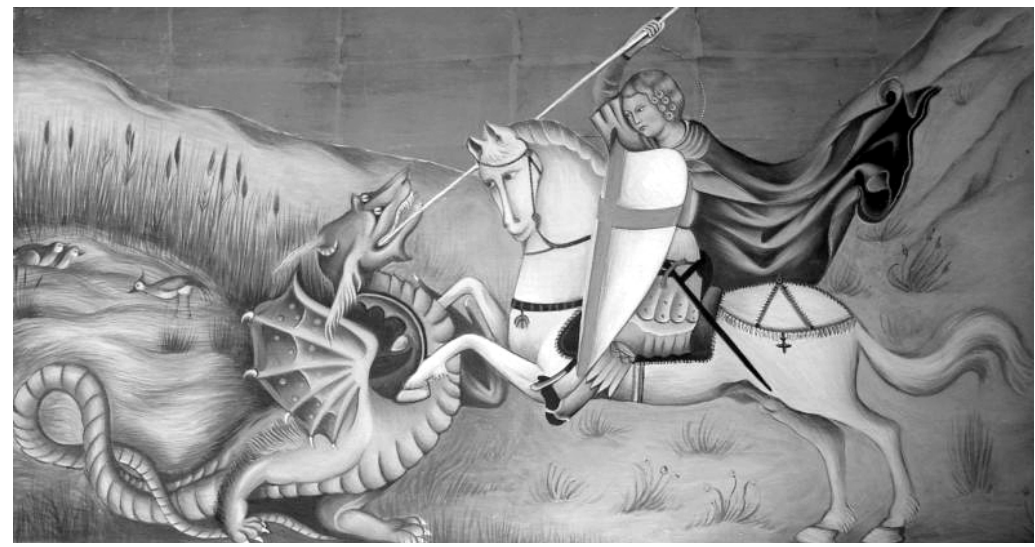

Particolare del San Giorgio e il drago ripreso da codice miniato, Città del Vaticano, Archivio Capitolare di San Pietro, Roma. 
B. Simone Martini (1340 c.), Minio del frontespizio del Virgilius cum notis Petrarcae; Milano, Biblioteca ambrosiana, ms. Ambrosiano S.P. 10.27.

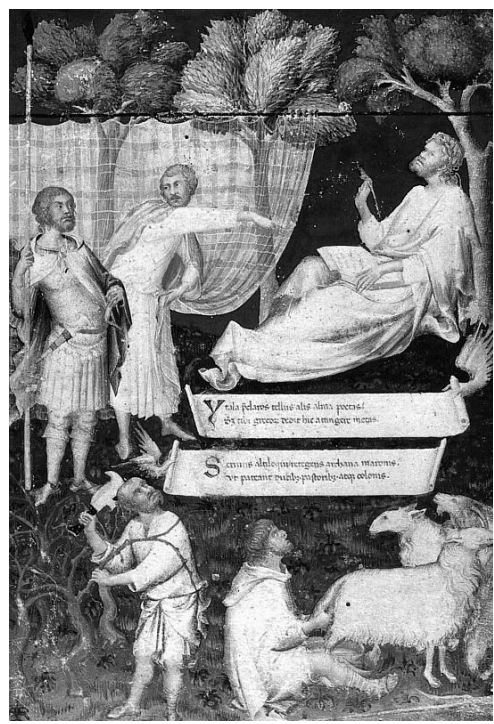

\section{Apendice II}

Carpentras - BM - ms. 0412 f. 020v, Le mont de Sagesse; Jean Lemaire de Belges, La Concorde des deux langages (16 secolo).

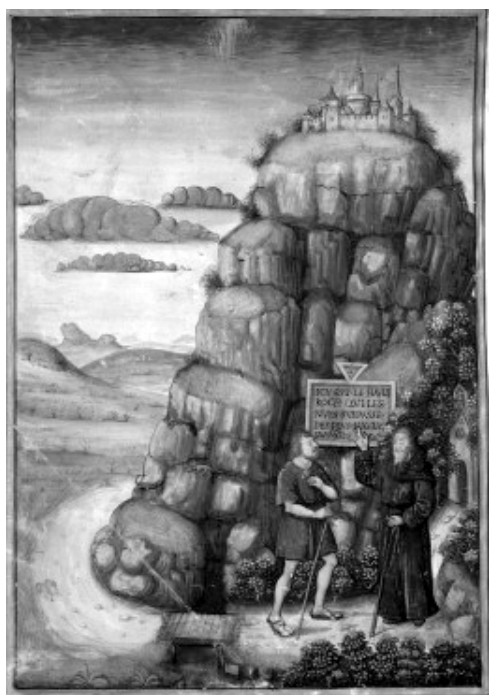




\section{Apendice III}

A. Autun - BM - ms. 0003, f. 175v, Pesce e uccello, Evangiles, (754-755 circa).

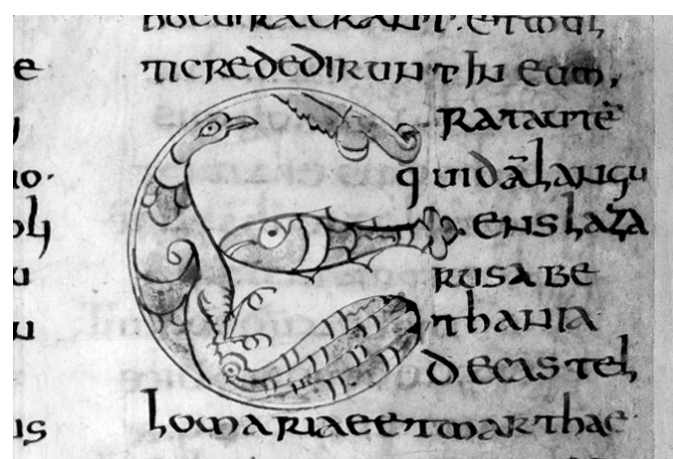

B. Besançon - BM - ms. 0140 (f. 001-189v) f. 045 Pesce e uccello, Salterio de Limoges (13 secolo, prima metà).

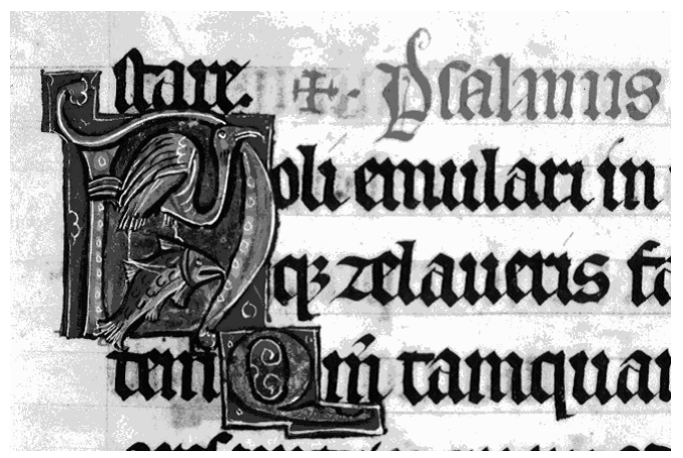

C. Rouen - BM - ms. 1381, f. 205v; Pesce e uccello; Vitae sanctorum (11 secolo).

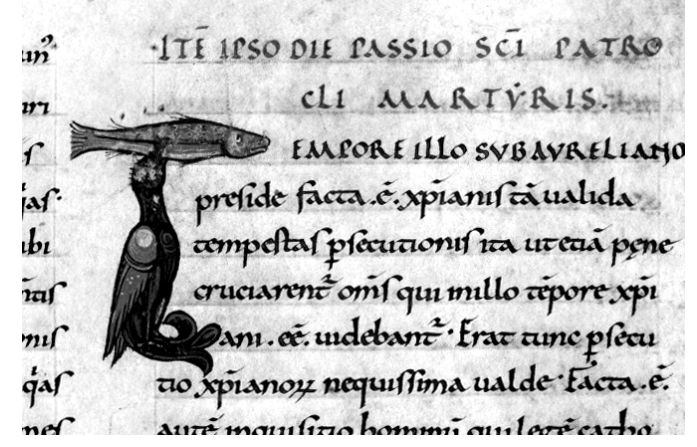


D. Chalon-sur-Saône - BM - ms. 0014 (f. 055-089), f. 075, Pesce e uccello; De Bestiis et aliis rebus (13 secolo).

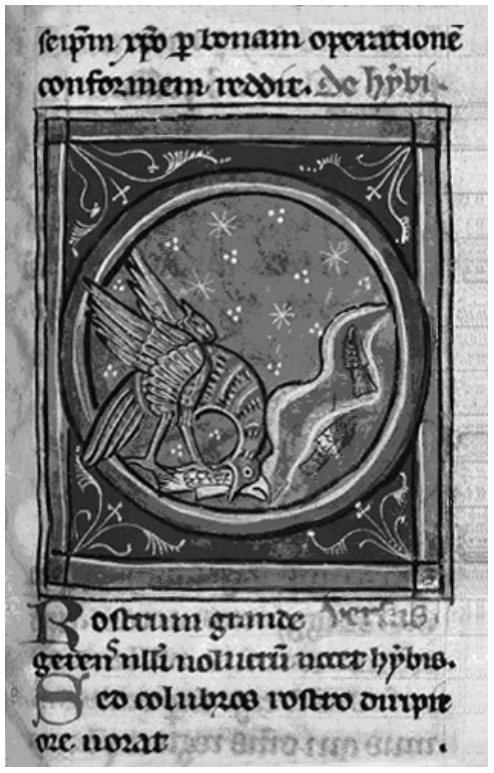

\section{Apendice IV}

A. Amiens - BM - ms. 0399, f. 144v, Grue, Bartholomaeus Anglicus, Le livre des proprietes des choses (1447).

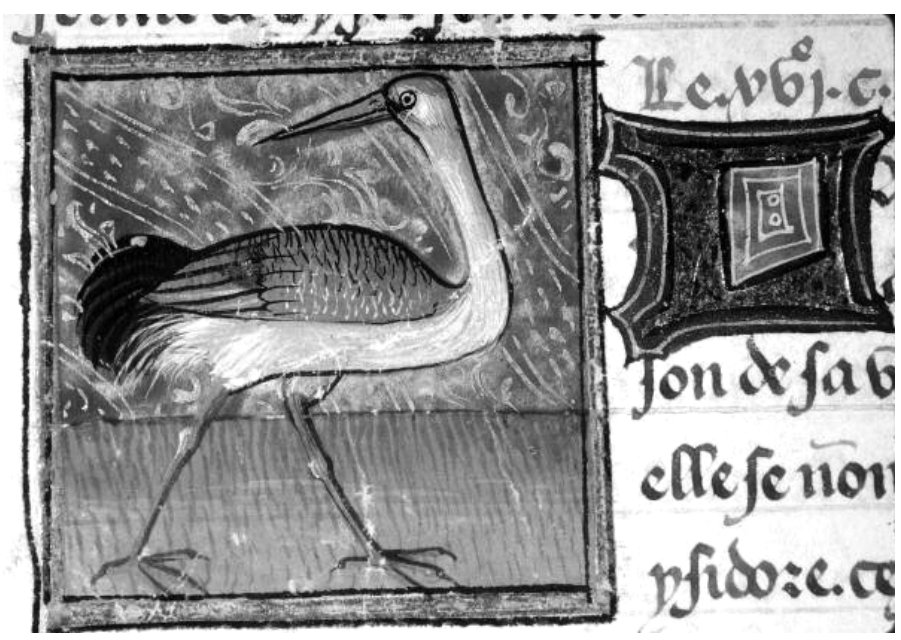


B. Marseille - BM - ms. $0111 \mathrm{f} .102 \mathrm{v}$; Gru gobant una pietra; Heures à l'usage de Thérouanne (1280-1290 circa).

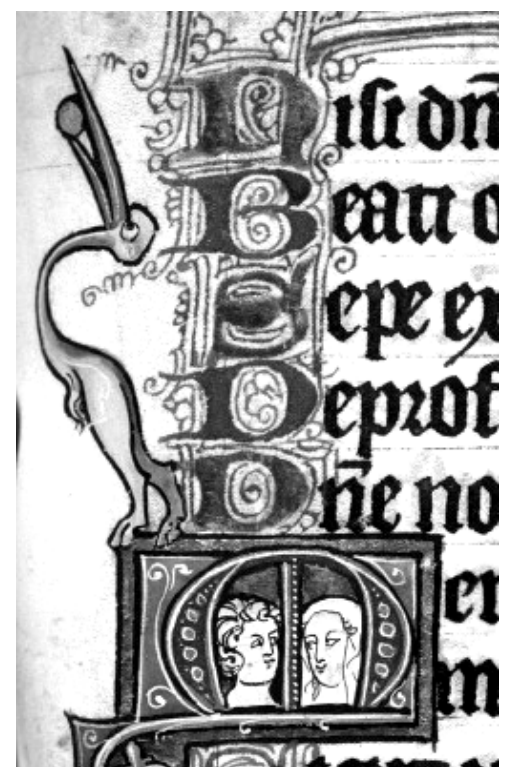

C. Dijon - BM - ms. 0526 f. 024v, Gru, Richard de Fournival; Guillaume de Lorris et Jean de Meung; Johannes de Sancto Maxentio, Recueil littéraire, (14 secolo (inizio); Francia; Nord; Picardie).

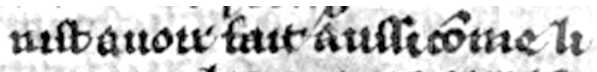
grus qu leg antoro gente gratte. 2, ntit genes thotu en catrule.coug toun an uelle

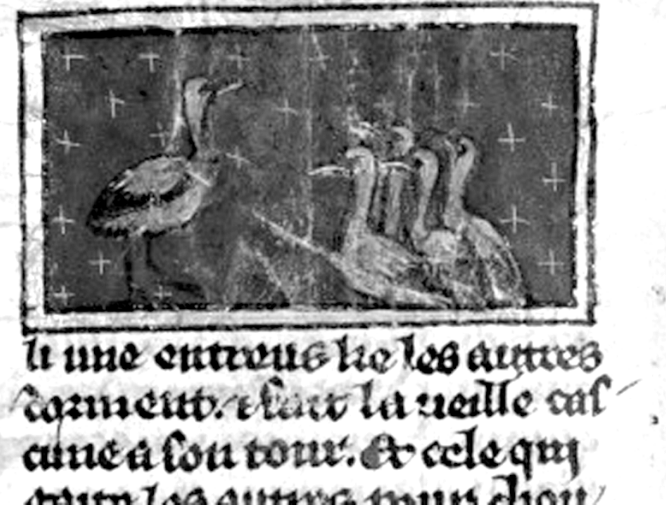

